H I G H L I G H T S

\section{Sir2 finds its voice in replication}

Reporting in Genes \& Development, Michael Weinreich and colleagues have identified a new role for the histone deacetylase Sir2 that is independent of its well-known roles in silencing - the negative regulation of the initiation of DNA replication in Saccharomyces cerevisiae.

The authors wanted to understand more about the assembly of pre-replicative complexes (pre-RCs), which establish replication competence. In S. cerevisiae, pre-RCs consist of Cdc6, the origin recognition complex, Cdt1 and the MCM helicase: once assembled they ultimately promote the formation of bidirectional replication forks.

They found that the deletion of silent information regulator SIR2 suppressed a $c d c 6-4$ replication-initiation mutant. But this was not indirect suppression through the loss of silencing or due to the loss of recombination. Using $c d c 6-4 \operatorname{sir} N 345 A$ double mutants - in which a single amino acid in the catalytic domain of Sir2 is mutated to abolish its deacetylase activity - Weinreich and colleagues found that it was the loss of enzymatic activity that suppressed the $c d c 6-4$ mutant. And this effect was not due to the stabilization of Cdc6 or because the requirement for $C D C 6$ was bypassed when SIR2 was deleted.

Deleting SIR2 rescued the initiation defect of $c d c 6-4$; it also rescued other initiation mutants, but only those affecting pre- $\mathrm{RC}$ assembly. In addition, in $c d c 6-4$ mutants, the deletion of SIR2 rescued the high rate of

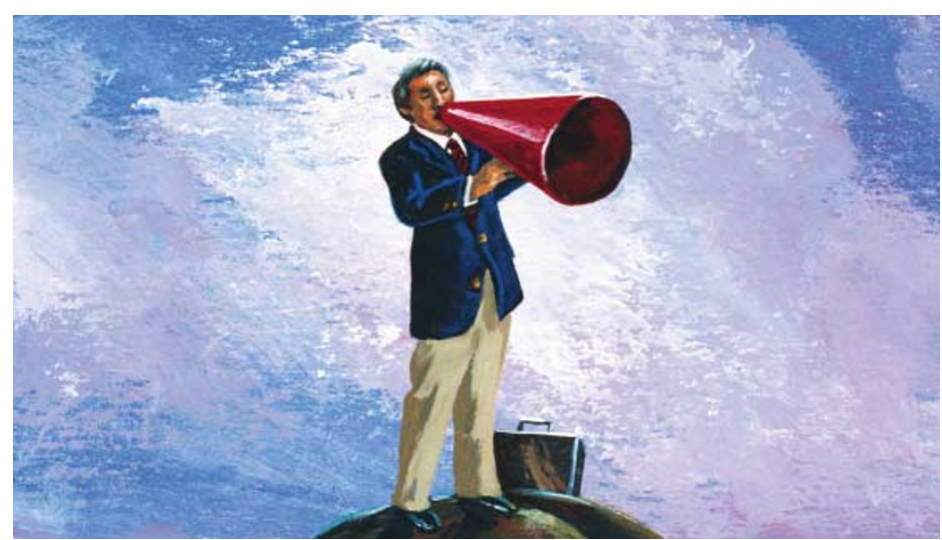

\section{STRUCTURE WATCH}

\author{
Bridging the gap
}

At present, there is a gap in our structural understanding of cellular complexes. We have comparatively low-resolution images of large cellular complexes - for example, electron microscopy (EM) images of the nuclear pore complex — and high-resolution structures of components of these complexes. As it seems that technical limitations will prevent us from obtaining highresolution structures of large complexes in the immediate future, how can we begin to bridge this gap? Russell and colleagues now describe one method in Science.

They modelled a large set of yeast protein-interaction data using known three-dimensional structures, and were able to obtain the most complete view of protein complexes so far and also to predict a structure-based network of crosstalk between complexes.

Furthermore, they screened complexes using EM to obtain lowresolution images that helped them to assemble and confirm their models. Of 102 complexes, at least 54 partial models could be obtained. And, for 29 of these - including the exosome, RNA polymerase II and a 3'-messenger-RNA-degradation complex the results revealed atomic details that would not readily have been seen by homology. These models have the potential to generate hypotheses that can be further tested, for example, by mutagenesis. In addition, as each new structure that becomes available can help us to understand numerous interactions, "The complex predictions and the associated network will ... improve exponentially as the numbers of structures and interactions increase, providing an ever more complete molecular anatomy of the cell."

REFERENCE Aloy, P. et al. Structure-based assembly of protein complexes in yeast. Science 303, 2026-2029 (2004)

\section{A new state of STAT}

Cytoplasmic STAT (signal transducer and activator of transcription) proteins are tyrosine phosphorylated as a result of the ligand-induced activation of cell-surface receptors. They then dimerize through reciprocal phosphotyrosine-SH2-domain interactions and translocate to the nucleus, where they regulate target-gene expression. Crystal structures are available for the core regions of mammalian STAT1 and STAT3 in their DNA-bound forms, but important questions still remain about the structures of non-DNA-bound STATs. Now, though, in Molecular Cell, Müller and co-workers present the $2.7-\AA$ crystal structure of the core region of a tyrosine phosphorylated, homodimeric Dictyostelium discoideum STAT (STATa) in its non-DNA-bound form.

Like STAT1 and STAT3, STATa has four domains, but the orientation of these domains is different - for example, the orientation of the coiled-coil domain is inverted. Dimerization of STATa is mediated by both phosphotyrosine-SH2-domain interactions and direct SH2-domain interactions, and the STATa dimer has an extended, 'open' structure that is very different to the 'closed' structure of the DNA-bound STAT1 and STAT3 dimers. This indicates how the non-DNA-bound and DNA-bound forms might be differentially recognized by factors that regulate STAT signalling, and implies that a large conformational change occurs on target recognition. In addition, this work has provided insights into the nuclear export, evolution and diversity of STAT proteins.

REFERENCE Soler-Lopez, M. et al. Structure of an activated Dictyostelium STAT in its DNA-unbound form. Mol. Cell 13, 791-804 (2004) 